\title{
Re: Post-Thaw Recovery of Rare or Very Low Concentrations of Cryopreserved Human Sperm
}

\author{
Kathrins $\mathrm{M}^{1}$, Abhyankar $\mathrm{N}^{2}$, Shoshany $\mathrm{O}^{2}$, Liebermann $\mathrm{J}^{3}$, Uhler $\mathrm{M}^{3}$, Prins $\mathrm{G}^{2}$, Niederberger $\mathrm{C}^{2}$ \\ ${ }^{1}$ Brigham and Women's Hospital, Division of Urology, Boston, USA \\ ${ }^{2}$ University of Illinois, Department of Urology, Chicago, USA \\ ${ }^{3}$ Fertility Centers of Illinois, Chicago, USA
}

Fertil Steril 2017;107:1300-1304. doi: 10.1016/j.fertnstert.2017.04.016.

\section{EDITORIAL COMMENT}

In men with azoospermia due to spermatogenic dysfunction (ASD) or men with very low sperm count "severe oligospermic men", sperm cryopreservation is very important back-up to save these sperms for future in vitro fertilization procedures. However, technical difficulties with cryopreservations are still a problem for men with very low sperm count. The most detrimental outcome of cryopreservation of very low concentrations of sperm is the absence of identifiable sperm after thawing. In this study, the authors investigated the cases of failure to retrieve any sperm after thawing of low concentrations of cryopreserved sperm. In this retrospective study, 55 men [83 intracytoplasmic sperm injection (ICSI) cycles] who met the inclusion criteria were included. Five different couples underwent 5 ICSI cycles (6.0\%) however, no sperm were identified after thawing. Four of these cases (8.5\%) were in the group of "rare sperm only" and one (2.8\%) in the group of $<100,000$ total sperm. It was observed that the ability to locate sperm after thawing was not statistically significantly associated with the pre-cryopreservation parameters and sperm source. The findings documented in this study could be valuable information for couples suffering from ASD who are in the process of decision making whether to start ovulation induction before or after surgical sperm retrieval.

Emre Bakırcıoğlu, MD

๑Copyright 2017 by the Association of Urological Surgery / Journal of Urological Surgery published by Galenos Publishing House. 